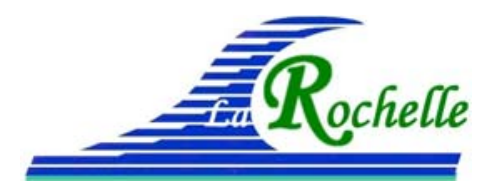

XVèmes Journées Nationales Génie Côtier - Génie Civil

La Rochelle, 29 au 31 mai 2018

DOI:10.5150/jngcgc.2018.043 C Editions Paralia CFL



\title{
Évaluation des mouvements verticaux du sol et des tendances relatives du niveau de la mer le long du littoral de Brest (France) : Apport de la télédétection.
}

\section{Cyril POITEVIN ${ }^{1}$, Guy WÖPPELMANN ${ }^{1}$, Daniel RAUCOULES ${ }^{2}$, Gonéri LE COZANNET ${ }^{2}$, Laurent TESTUT ${ }^{1,3}$}

1. LIENSs, Université de La Rochelle, UMR 7266 CNRS, 2 rue de Olympe de Gouges, 17000 La Rochelle, France.

contact-lienss@univ-lr.fr

2. BRGM,

3 Avenue Claude Guillemin, 45060 Orléans Cédex, France.

contact-brgm@brgm.fr

3. LEGOS, Université de Toulouse, CNES, CNRS, IRD, UPS, 18 Avenue Edouard Belin, 31401 Toulouse Cédex 9, France.

\section{Résumé :}

Selon l'influence des mouvements verticaux côtiers, l'observation de la montée du niveau de la mer induite par le changement climatique peut différer d'un littoral à un autre. Prendre en compte la variabilité spatiale de ces mouvements est donc primordial pour évaluer au mieux les risques liés au changement climatique le long du littoral. La mesure des mouvements verticaux du sol à la côte est principalement assurée par les stations GPS (Global Positioning System) permanentes rattachées aux marégraphes mais les données récoltées sont souvent trop éparses pour caractériser l'étendue spatiale des mouvements terrestres. Une approche de télédétection comme l'InSAR (Interferometric Synthetic Aperture Radar) peut fournir des cartes de déformations superficielles sur de grandes étendues spatiales et peut apporter une solution complémentaire aux données GPS. Dans un premier temps, l'apport de l'InSAR a été évalué sur la zone de Brest (France), zone d'intérêt face à l'importance de la série marégraphique séculaire de Brest pour l'étude du changement climatique à l'échelle globale. Les résultats indiquent que la zone de Brest est majoritairement très stable excepté le long des zones portuaires de la ville où des subsidences de faible ampleur (valeurs moyennes inférieures à $1 \mathrm{~mm} / \mathrm{an}$ ) sont remarquables. Dans un deuxième temps, les tendances relatives du niveau la mer ont été reconstituées le long du linéaire côtier de Brest en combinant les données InSAR et GPS aux données d'altimétrie spatiale de la zone, illustrant ainsi la variabilité spatiale de ces tendances.

Mots-clés :

InSAR, Mouvements verticaux, Niveau de la mer, Littoral, Altimétrie radar, Brest. 


\section{Thème 3 - Instrumentation, mesures, imagerie et télédétection}

\section{Introduction}

Pour évaluer de manière complète les variations du niveau de la mer observées à la côte et les risques associées à la montée du niveau de la mer sur le littoral, il est nécessaire de déterminer précisément les mouvements verticaux côtiers de la terre solide. Une mesure précise de ces mouvements sur le littoral est notamment apportée par les stations GPS co-localisées aux marégraphes dont l'objectif est de déterminer la composante terrestre du signal enregistré par les marégraphes à une précision inférieure au millimètre par an, précision nécessaire dans l'étude des tendances du niveau de la mer sur le long terme (WÖPPELMANN \& MARCOS, 2016). En effet, les marégraphes étant installés sur la côte, ils mesurent à la fois les variations du niveau de la mer et les mouvements verticaux du socle sur lequel ils reposent et fournissent ainsi une mesure directe des tendances relatives du niveau de la mer. L'installation des stations GPS est néanmoins souvent trop éparse pour rendre compte de la variation spatiale des mouvements verticaux du sol le long de la côte. Il est cependant difficile d'estimer l'emprise spatiale des tendances relatives du niveau de la mer mesurées par les marégraphes, principalement en raison de cette variabilité spatiale locale, liée à de nombreux processus de mouvement de la terre solide (tectonique, volcanique, compaction de sédiment, extraction de fluides souterrains) qui peuvent être enregistrés. Une précédente étude (BROOKS et al., 2007) a démontré qu'il était possible d'utiliser l'InSAR (Interferometric Synthetic Aperture Radar) en combinaison avec des données GPS et marégraphiques pour étendre spatialement l'information du marégraphe le long $\mathrm{du}$ littoral. Dans cette étude, nous proposons d'utiliser plutôt l'information de l'altimétrie radar embarquée sur satellite en complément des données GPS et InSAR pour déterminer comment varie le niveau marin relatif le long de la côte. Nous revenons sur les détails de la méthode InSAR, que nous avons évaluée sur la zone de Brest (France) où des questions persistent sur les mouvements verticaux effectivement en présence (soulèvement (LENÔTRE et al., 1999), subsidence (GOSLIN et al., 2015), stabilité (SANTAMARIA-GOMEZ et al., 2017)).

\section{Matériels et méthodes}

\subsection{Zone d'étude}

Cette étude a été menée sur la zone de Brest (France) (voir Figure 1) dont la connaissance précise des mouvements verticaux est primordiale face à l'apport de la série marégraphique de Brest dans l'étude des tendances globales du niveau de la mer (WÖPPELMANN et al., 2008), notamment dans un programme international comme celui du Global Sea Level Observing System (GLOSS) (INTERGOVERNMENTAL OCEANOGRAPHIC COMMISSION, 2012). Actuellement, et comme recommandé par le programme GLOSS, une station GPS permanente (BRST) est installée à proximité du marégraphe. D'après l'analyse des mesures du GPS, la zone serait stable avec une 


\section{XVèmes Journées Nationales Génie Côtier - Génie Civil \\ La Rochelle, 29 au 31 mai 2018}

vitesse verticale GPS de $0.01 \pm 0.11 \mathrm{~mm} /$ an entre 1998 et 2014 (SANTAMARIAGOMEZ et al., 2017).

Le sous-sol de la zone est principalement constitué de granit et de gneiss datant des orogénèses cadomienne (650-550 Ma) et varisque (420-290 Ma) (voir Figure 1). Le littoral de Brest est principalement caractérisé par la présence de remblais mis en place suite au développement des infrastructures portuaires de la ville. Ces couches de remblais peuvent atteindre des profondeurs de $20 \mathrm{~m}$ (SAUSSAC et al., 2015) et sont constituées de matériaux sablo-argileux hétérogènes.

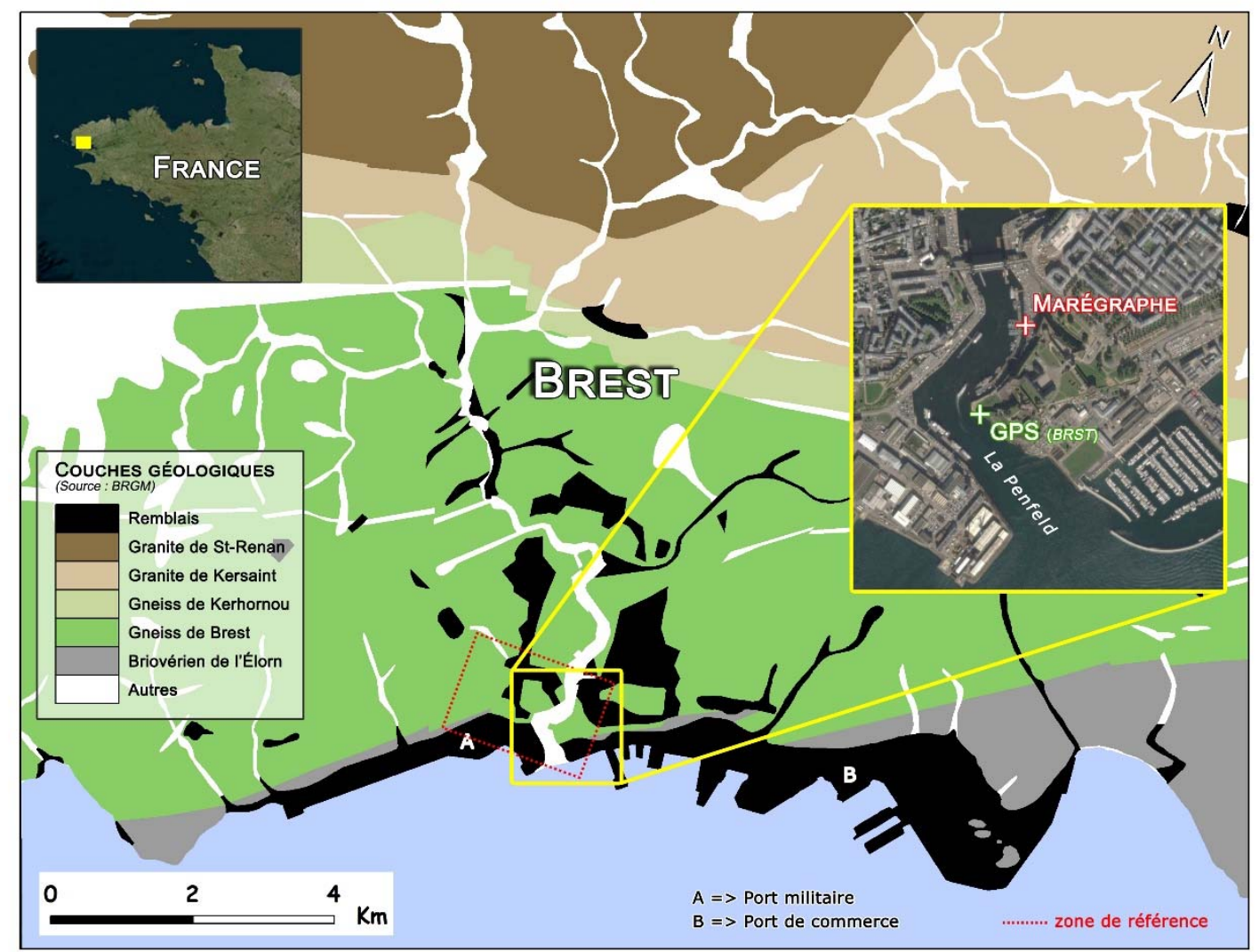

Figure 1. Structure géologique de la zone d'étude avec un zoom sur la zone où sont situés les instruments géodésiques d'intérêts.

\subsection{Analyse InSAR}

Dans le cadre de cette étude, l'analyse InSAR a été réalisée avec le logiciel StaMPS/MTI (Stanford Method for Persistent Scatterers) (HOOPER et al., 2004 ; HOOPER, 2008). Ce logiciel s'appuie sur une approche Multi-Temporal InSAR (MTI) dont l'objectif est de déterminer les pixels, ou PS (Persistent Scatterers), dont le signal retour démontre des caractéristiques physiques stables dans le temps, i.e. sur plusieurs images SAR (Synthetique Aperture Radar) d'une même zone. En se focalisant sur les pixels les plus cohérents, cette approche permet ainsi de détecter des mouvements 


\section{Thème 3 - Instrumentation, mesures, imagerie et télédétection}

terrestres à une précision de l'ordre du millimètre par an, contrairement à l'approche InSAR classique dont la précision ne dépasse pas le centimètre par an (Plus de détails dans CROSETTO et al., 2016). StaMPS/MTI présente, de plus, l'avantage de détecter une densité importante de PS en milieu naturel.

Les images SAR de cette étude ont été extraites des missions satellitaires ERS-1/2 et ENVISAT dont les périodes d'acquisition présentent une adéquation temporelle intéressante avec les données GPS (1998-2014) et d'altimétrie satellitaire (1993-2014) disponibles sur la zone. 48 images SAR proviennent des archives des missions ERS-1/2, de mai 1992 à novembre 2000, et 19 images SAR proviennent des archives de la mission ENVISAT, de décembre 2002 à mars 2008. Pour limiter le temps de calcul et les ressources informatiques nécessaires, chaque image SAR a été découpée selon une zone recentrée sur Brest. Une zone de référence (voir Figure 1), nécessaire aux traitements InSAR, a été calibrée autour du GPS BRST du fait de la stabilité du sol indiquée par celui-ci.

\subsection{Combinaison InSAR \& Altimétrie}

Selon l'expression (1) (WÖPPELMANN \& MARCOS, 2016), les tendances relatives du niveau de la mer ( $\left.\mathrm{SL}_{\text {relative }}\right)$ peuvent-être déterminées à partir des données de tendances absolues du niveau de la mer provenant de l'altimétrie $\left(\mathrm{SL}_{\text {absolute }}\right)$ combinées à des données de mouvements verticaux côtiers du socle terrestre $\left(\mathrm{VLM}_{\text {absolute }}\right)$.

SLrelative $=$ SLabsolute - VLMabsolute

Les grandeurs "absolues" de la relation (1) sont déterminées par rapport à l'ellipsoïde du repère de référence terrestre centré au centre de masses de la terre. Les résultats InSAR sont calculés par rapport à la zone de référence (voir Figure 1). Il convient donc de rapporter les données InSAR dans le même référentiel terrestre que les données altimétriques pour combiner les deux jeux de données. La meilleure solution pour obtenir des données InSAR absolues est d'attacher rigoureusement les PS situés dans la zone de référence à la station GPS la plus proche à l'aide de points de contrôle au sol (ou PS artificiels) tels que des "corner-reflectors" ou des transpondeurs radar actifs (MAHAPATRA et al., 2017). Si aucun PS artificiel n'est disponible sur la zone de mesure, il est possible de déterminer un plan de transformation des données InSAR en minimisant la différence entre les mouvements terrestres verticaux mesurés par InSAR et ceux mesurés par plusieurs stations GPS (SHENNAN et al., 2015). Avec seulement une station GPS disponible sur la zone d'étude et aucun PS artificiel disponible, ces méthodes ne peuvent être appliquées dans cette étude. Néanmoins, une valeur de transformation simple a été déterminée en prenant la différence entre la valeur de déplacement vertical estimé par le GPS BRST et une moyenne des valeurs de déplacement vertical obtenue avec les PS environnants. 


\section{XVèmes Journées Nationales Génie Côtier - Génie Civil \\ La Rochelle, 29 au 31 mai 2018}

\section{Résultats et discussion}

\subsection{Analyse InSAR}

Les résultats de l'analyse InSAR pour les périodes 1992-2000 et 2002-2008 sont présentés dans la Figure 2. Pour chaque jeu de données SAR, les données ont été spatialement agrégées dans une maille régulière en calculant la moyenne pondérée des valeurs de déplacements contenus dans une même cellule de la maille pour obtenir une information continue et lisse des déplacements sur la zone d'étude. Les calculs ont été réalisés sur des cellules comprenant au moins trois valeurs de déplacement, i.e. au moins trois PS. La résolution des cellules a été définie à $300 \mathrm{~m}$ pour obtenir un compromis entre une densité suffisante de cellules avec une information de déplacement vertical et une bonne restitution des zones à faible densité de PS. Les valeurs de déplacement sont obtenues selon l'axe de visé du satellite et sont exprimées de telle sorte que les valeurs négatives correspondent à des valeurs de subsidence. L'angle de visée des satellites ERS-1/2 et ENVISAT pour l'acquisition des images radars étant faible $\left(23^{\circ}\right)$, les valeurs mesurées dans l'axe de visée du satellite sont environ 2.3 fois plus sensible à la composante verticale qu'à la composante horizontale (RAUCOULES et al., 2013), les valeurs obtenues sont donc considérés comme principalement liées à la composante verticale du déplacement.

Avec des valeurs de moyenne pondérée comprises entre $-0.7 \mathrm{~mm} /$ an et $0.7 \mathrm{~mm} /$ an pour la majorité des cellules ( $81 \%$ pour ERS-1/2 et $85 \%$ pour ENVISAT), les deux cartes de de la Figure 2 indiquent que la zone est globalement stable. Cette stabilité est particulièrement observable sur le centre-ville de Brest et notamment dans la zone où la station GPS BRST et le marégraphe sont localisés.

Des zones de subsidence côtière de plus de $1 \mathrm{~mm}$ /an sont observables sur chaque jeu de données. Aucune relation spatiale n'a été mise en évidence en superposant les résultats InSAR avec la structure géologique de la zone (Figure 1). Néanmoins l'emprise spatiale des remblais côtiers (Figure 1) correspond à l'emprise spatiale des subsidences suggérant ainsi un lien entre les subsidences et les activités portuaires. L'origine de ces subsidences est probablement liée à des phénomènes de compaction des remblais mis en place successivement au cours du $\mathrm{XX}^{\text {ème }}$ siècle pour étendre et adapter la capacité portuaire de la ville de Brest aux évolutions économiques et industrielles. Malgré une bonne cohérence spatiale des déplacements observés entre les deux jeux de données InSAR, une zone située au nord de la zone d'étude montre des valeurs de soulèvement d'environ $1.5 \mathrm{~mm} /$ an pour les données issues d'ERS-1/2 tandis que la même zone montre des valeurs de subsidence d'environ $-1 \mathrm{~mm} / \mathrm{an}$ pour les données issues d'ENVISAT. Ces résultats contradictoires peuvent indiquer un changement de comportement de la zone entre les deux périodes analysées. En effet, cette zone est potentiellement concernée par des risques géologiques superficiels tels que des 
Thème 3 - Instrumentation, mesures, imagerie et télédétection

glissements de terrains de faible intensité ou des phénomènes de gonflement des argiles (www.georisques.gouv.fr).

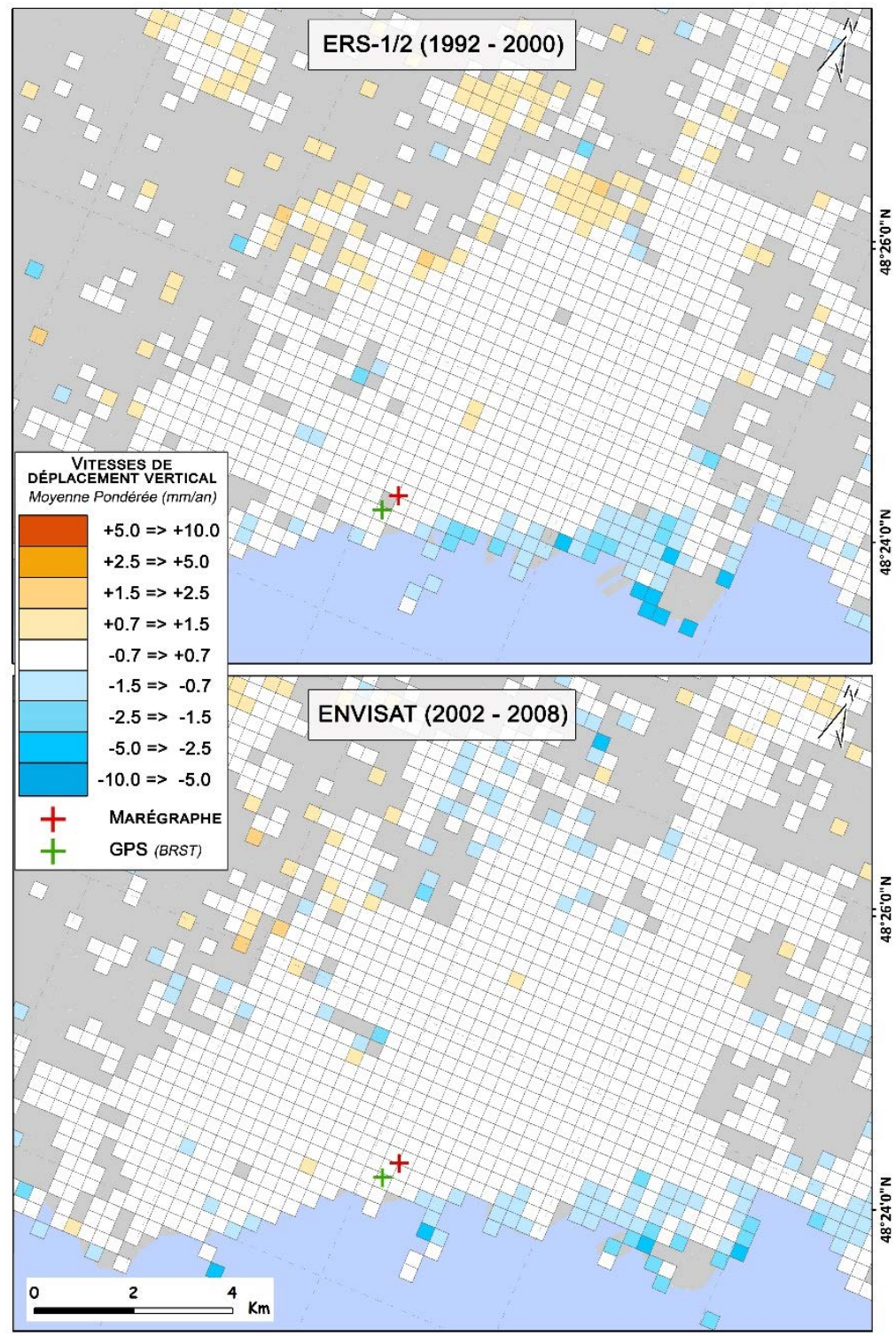

Figure 2. Cartes des vitesses de déplacement vertical du sol obtenues avec StaMPS/MTI avec les données d'ERS-1/2 (1992-2000) et d'ENVISAT (2002-2008).

\subsection{Combinaison InSAR \& Altimétrie satellitale}

La Figure 3 présente les tendances relatives du niveau de la mer obtenues par la soustraction des hauteurs du niveau de la mer mesurée par l'altimétrie radar satellitale et les mouvements verticaux côtiers obtenus par l'analyse InSAR et GPS. La combinaison des données a été appliquée uniquement sur les cellules localisées sur le littoral de Brest ce qui représente environ $23 \mathrm{~km}$ de linéaire côtier. 


\section{XVèmes Journées Nationales Génie Côtier - Génie Civil \\ La Rochelle, 29 au 31 mai 2018}

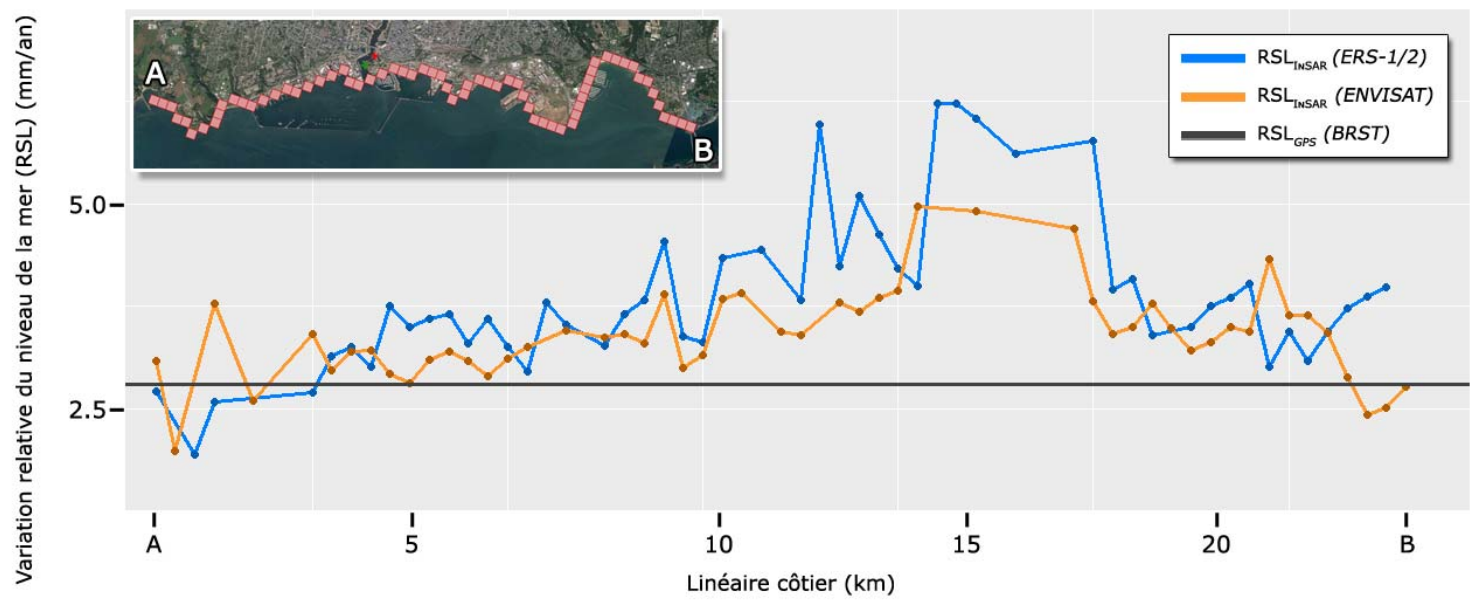

Figure 3. Profils de tendances relatives du niveau de la mer pour chaque jeu de données InSAR et GPS combinées avec des données d'altimétrie radar satellitales entre deux positions (A et B) le long de la côte de Brest.

Ces résultats démontrent l'intérêt de l'approche pour obtenir une information plus riche et détaillée des variations relatives du niveau de la mer le long du littoral. Il est possible avec cette approche de déterminer efficacement les zones d'intérêts où la détermination précise du niveau relatif de la mer est un enjeu important face aux risques de la montée du niveau de la mer. Une attention particulière doit néanmoins être portée à l'expression des données InSAR dans un repère terrestre géocentrique.

\section{Conclusions}

L'analyse InSAR de la zone de Brest apporte de nouvelles connaissances sur la variabilité des mouvements verticaux qui affectent la région. Les résultats InSAR montrent que la zone de Brest est majoritairement stable, ce qui confirme l'observation ponctuelle de la station GPS BRST, néanmoins ils révèlent également deux zones de subsidence au niveau des zones portuaires. L'identification de ces subsidences d'origines vraisemblablement anthropiques démontre que la représentativité de la mesure GPS est spatialement limitée et justifie l'apport d'une méthode complémentaire comme l'InSAR. Néanmoins la précision des données InSAR n'atteint pas encore celle des résultats GPS. De plus, l'InSAR a un caractère relatif et ne peut donc pas être considéré comme une méthode de remplacement, mais plutôt de complément à la méthode GPS. La combinaison des données InSAR à des données d'altimétrie radar satellitale apporte une solution intéressante dans l'identification des zones côtières les plus susceptibles aux impacts de la montée du niveau de la mer. Cette combinaison nécessite des développements complémentaires mais présente déjà une solution intéressante dans la résolution des tendances relatives du niveau de la mer sur les littoraux où aucune station marégraphique ne serait disponible. 
Thème 3 - Instrumentation, mesures, imagerie et télédétection

\section{Remerciement}

Cette étude a bénéficié d'un support financier du CNES (Centre National d'Étude Spatiales) par l'intermédiaire d'une bourse TOSCA. Les données issues des missions satellitaires ERS-1/2 et ENVISAT ont été téléchargées gratuitement avec le support de l'ESA (European Space Agency) à travers le projet Cat-1/19372.

\section{Références bibliographiques}

BROOKS B. A., MERRIFIELD M. A., FOSTER J., WERNER C. L., GOMEZ F., BEVIS M., GILL S. (2007). Space geodetic determination of spatial variability in relative sea level change, Los Angeles basin. Geophys. Res. Lett. Vol. 34(01). https://doi.org/10.1029/2006GL028171

CROSETTO M., MONSERRAT O., CUEVA-GONZALEZ M., DEVANTHERY N., CRIPPA B. (2016). Persistent scatterer interferometry: A review. ISPRS Journal of Photogrammetry and Remote Sensing, Vol. 115, pp 78-89. https://doi.org/10.1016/j.isprsiprs.2015.10.011

GOSLIN J., VAN VLIET-LANOE B., SPADA G., BRADLEY S., TARASOV L., NEILL S., SUANEZ S. (2015). A new Holocene relative sea-level curve for western Brittany (France): Insights on isostatic dynamics along the Atlantic coasts of northwestern Europe. Quarternary Science Reviews, Vol. 129, pp 341-365. https://doi.org/10.1016/j.quascirev.2015.10.029

HOOPER A., ZEBKER H., SEGALL P., KAMPES B. (2004). A new method for measuring deformation on volcanoes and other natural terrains using InSAR persistent scatterers. Geophys. Res. Lett. Vol. 31(23), 5 p. https://doi.org/10.1029/2004GL021737

HOOPER A. (2008). A multi-temporal InSAR method incorporating both persistent scatterer and small baseline approaches. Geophys. Res. Lett. Vol. 35(16), 5 p. https://doi.org/10.1029/2008GL034654

INTERGOVERNMENTAL OCEANOGRAPHIC COMISSION (IOC) (2012). Global Sea Level Observing System (GLOSS): Implementation Plan - 2012, UNESCO/IOC, 41p., IOC Technical Series, No.100.

MAHAPATRA P., VAN DER MAREL H., VAN LEIJEN F., SAMIEI ESFAHANY S., KLEES R., HANSSEN R. (2017). InSAR datum connection using GNSS-augmented radar transponders. Journal of Geodesy. https://doi.org/10.1007/s00190-017-1041-y

LENÔTRE N., THIERRY P., BLANCHIN R., BROCHARD G. (1999). Current vertical movement demonstrated by comparative levelling in brittany (northwestern france). Tectonophysics, Vol. 301(3-4), pp 333-344. https://doi.org/10.1016/S0040-1951(98)00228-5

RAUCOULES D., LE COZANNET G., WÖPPELMANN G., DE MICHELE M., GRAVELLE M., DAAG A., MARCOS M. (2013). High nonlinear urban ground motion in Manila (Philippines) from 1993 to 2013 observed by DInSAR: Implications for sea-level measurement. Remote Sensing of Environment, Vol. 139, pp 386-397. https://doi.org/10.1016/j.rse.2013.08.021 


\section{XVèmes Journées Nationales Génie Côtier - Génie Civil \\ La Rochelle, 29 au 31 mai 2018}

SANTAMARIA-GOMEZ A., GRAVELLE M., DANGENDORF S., MARCOS M., SPADA G., WÖPPELMANN G. (2017). Uncertainty of the 20th century sea-level rise due to vertical land motion errors. Earth and Planetary Science Letters, Vol. 473, pp 24-32. https://doi.org/10.1016/j.epsl.2017.05.038

SAUSSAC L., MOAL E., GANDIHON A. (2015). Brest Military Harbour: Treatment of liquefaction risk by ballasted columns behind a dock wall. Travaux Maritimes et Fluviaux, Vol. 914, pp 70-76.

SHENNAN I., LONG A.J., HORTON B.P. (2015). Handbook of Sea-Level Research, First Edition. American Geophysical Union. John Wiley \& Sons, Ltd.

WÖPPELMANN G., POUVREAU N., COULOMB A., SIMON B., WOODWORTH P.L. (2008). Tide gauge datum continuity at Brest since 1711: France's longest sea level record. Geophys. Res. Lett. Vol. 35(22). https://doi.org/10.1029/2008GL035783

WÖPPELMANN G., MARCOS M. (2016). Vertical land motion as a key to understanding sea level change and variability. Reviews of Geophysics, Vol. 54(1), pp 64-92. https://doi.org/10.1002/2015RG000502 
Thème 3 - Instrumentation, mesures, imagerie et télédétection 